\title{
The Asiago Supernova Catalogue - 10 years after ${ }^{\star}$
}

\author{
R. Barbon ${ }^{1}$, V. Buondí ${ }^{1}$, E. Cappellaro ${ }^{2}$, and M. Turatto ${ }^{2}$ \\ 1 Osservatorio Astrofisico dell'Università di Padova, I-36012 Asiago, Italy \\ 2 Osservatorio Astronomico di Padova, vicolo dell'Osservatorio 5, I-35122 Padova, Italy
}

Received May 7; accepted August 6, 1999

\begin{abstract}
Ten years after the publication of the previous release, we present a new edition of the Asiago Supernova Catalogue updated to December 31, 1998 and containing data for 1447 supernovae and their parent galaxies. In addition to the list of the data for a large number of new $\mathrm{SNe}$, we made an effort to search the literature for new information on past $\mathrm{SNe}$ as well. We also tried to update and homogenize the data for the parent galaxies. To allow a global view of the Catalogue, a few descriptive figures and a summary table are reported. The present Catalogue is intended as a large and modern database for statistical studies on the supernova phenomenon.
\end{abstract}

Key words: supernovae and supernova remnants: general - surveys - galaxies: general — galaxies: stellar contents of

\section{Introduction}

The interest of the scientific community on supernovae (SNe) has enormously increased in the recent years for several reasons. The advances in the understanding of the SN phenomena obtained with the intensive study of nearby SNe, first of all SN 1987A, have raised new more fundamental questions with regard to progenitor evolution, explosion mechanism and nucleosynthesis. In addition, the calibration of the absolute magnitudes of a few SNIa obtained using the Cepheid variables found in their parent galaxies (Saha et al. 1999, and references therein), and the discovery of empirical relations between the absolute magnitudes at maximum and the shape of the light curves of SNIa (Phillips 1993; Riess et al. 1996) have renewed the interest for the utilization of SNIa as distance indicators

Send offprint requests to: R. Barbon

* The complete Catalogue is only available in electronic form at the CDS via anonymous ftp to cdsarc.u-strasbg.fr (130.79.128.5) or via http://cdsweb.ustrasbg.fr/Abstract.html up to cosmological distances. Other exciting advances are expected for the association of some SNe with the mysterious GRBs. Such wide interest has triggered new, deep SN searches which, in a few years have doubled the number of SN discoveries.

The history of the Asiago SN catalogue began in 1984 with the publication of data for 568 objects (Barbon et al. 1984). This was compiled starting from the Palomar Supernova Master List which has since 1958 from time to time appeared in the literature (Zwicky 1958 and 1965; Kowal \& Sargent 1971; Sargent et al. 1974). During the same period two other SN listing have been published, by Karpowicz \& Rudnicki (1968) and by Flin et al. (1979), the latter giving also the complete bibliography for each object. The 1984 Asiago SN Catalogue was superseded by a new edition in 1989 (Barbon et al. 1989, [ASC89]) which listed information for the 661 supernovae discovered up to December 31, 1988.

More recently, van den Bergh (1994) published a list containing the 203 supernovae discovered between January 1, 1989 and April 3, 1994 and a Catalogue of extragalactic Supernovae, complete up to 1993, was published in volume $\mathrm{V}$ of the General Catalogue of Variable Stars (Samus 1995).

In the last few years we made available through the WEB at the URL athena.pd.astro.it/ supern/ a running SN list which has been widely utilized in the literature. Other supernova listings are available electronically, e.g. the list at the CBAT (www.harvard.edu/iau/lists/Supernovae. $h t m l$ ), and that at Sternberg Astronomical Institute (www.sai.msu.su/cgi-bin/wdb-p95/sn/sncat/form).

The many requests for a new, reliable edition give the motivation for the preparation of the present paper.

\section{The catalogue}

The new edition of the Asiago SN Catalogue lists data for $1447 \mathrm{SNe}$ and for their parent galaxies discovered up to 31 December, 1998. For the galaxy data we made large use of the Third Reference Catalogue of Bright Galaxies by de 
Table 3. Supernovae not included in the ASC89

\begin{tabular}{lll}
\hline $1945 \mathrm{~B}$ & $1975 \mathrm{U} ?$ & $1983 \mathrm{ab}$ \\
$1950 \mathrm{~N} ?$ & $1975 \mathrm{~V} ?$ & $1985 \mathrm{~S}$ \\
$1950 \mathrm{O} ?$ & $1976 \mathrm{O} ?$ & $1985 \mathrm{~T} ?$ \\
$1951 \mathrm{~J} ?$ & $1976 \mathrm{P} ?$ & $1986 \mathrm{P} ?$ \\
$1953 \mathrm{~J} ?$ & $1976 \mathrm{Q} ?$ & $1987 \mathrm{Q} ?$ \\
$1953 \mathrm{~K} ?$ & $1977 \mathrm{I} ?$ & $1987 \mathrm{R} ?$ \\
$1953 \mathrm{~L} ?$ & $1978 \mathrm{~J}$ & $1987 \mathrm{~S}$ \\
$1954 \mathrm{ac} ?$ & $1978 \mathrm{~K}$ & $1988 \mathrm{ac}$ \\
$1954 \mathrm{ad} ?$ & $1978 \mathrm{~L} ?$ & $1988 \mathrm{ad} ?$ \\
$1955 \mathrm{Q} ?$ & $1980 \mathrm{Q}$ & $1988 \mathrm{ae}$ \\
$1955 \mathrm{R} ?$ & $1982 \mathrm{Z} ?$ & $1988 \mathrm{af}$ \\
$1955 \mathrm{~S} ?$ & $1982 \mathrm{aa}$ & $1988 \mathrm{ag}$ \\
$1962 \mathrm{R} ?$ & & \\
\hline
\end{tabular}

Vaucouleurs et al. (1991, [RC3]) and of the LEDA ${ }^{1}$ and $\mathrm{NED}^{2}$ databases.

The format of the new edition follows that of ASC89 with some improvements. In particular, we have now included:

1. Accurate supernova positions;

2. Position angles of the major axes;

3. Morphological type code of the parent galaxies.

Accurate SN positions are mostly useful to compare observations at different wavelengths, from X-ray to radio, which in recent years had a large impact in SN research. Note that in the present Catalogue all coordinates are given at the 2000.0 epoch. Major axis position angles were introduced to study the position of the $\mathrm{SNe}$ within the galaxies and numerical morphological type code to facilitate the derivation of descriptive statistics.

Instead, we choose to drop the information on the parent galaxy luminosity classes because this information is available only for a small fraction (less than 20\%) of the objects. The galaxy integrated luminosity can be computed from the apparent magnitudes and distances of the galaxies.

To facilitate the consultation of the Catalogue, we present it with two different sortings: in Table 1 the list is arranged chronologically according to the date of SN discovery while in Table 2 the same data are listed in order of Right Ascension. follows:

In the tables, the content of the different columns is as

\footnotetext{
${ }^{1}$ LEDA Lyon-Meudon Extragalactic Database; www-obs.univ-lyon1.fr

${ }^{2}$ The NASA/IPAC Extragalactic Database (NED) is operated by the Jet Propulsion Laboratory, California Institute of Technology, under contract with the National Aeronautics and Space Administration.
}

Table 4. Objects which turned out not to be real supernovae

\begin{tabular}{lll}
\hline $1950 \mathrm{E}$ & $1986 \mathrm{~F}$ & $1991 \mathrm{~W}$ \\
$1956 \mathrm{C}$ & $1986 \mathrm{H}$ & $1991 \mathrm{ap}$ \\
$1967 \mathrm{~F}$ & $1987 \mathrm{E}$ & $1992 \mathrm{~W}$ \\
$1973 \mathrm{G}$ & $1987 \mathrm{H}$ & $1993 \mathrm{U}$ \\
$1893 \mathrm{H}$ & $1988 \mathrm{X}$ & $1993 \mathrm{~V}$ \\
$1985 \mathrm{~J}$ & $1990 \mathrm{C}$ & $1998 \mathrm{di}$ \\
$1986 \mathrm{D}$ & $1992 \mathrm{X}$ & \\
\hline
\end{tabular}

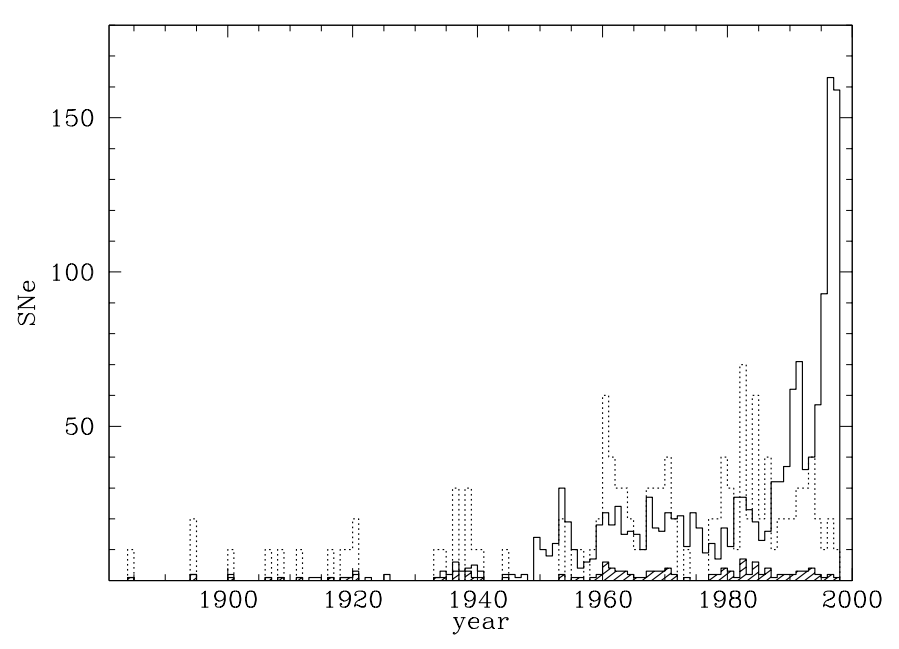

Fig. 1. Histogram of the number of SNe discovered per year. The shaded area refers to $\mathrm{SNe}$ with magnitude at maximum (or at discovery) brighter than 14 which, enlarged by a factor 10 , are also shown as dotted lines

1: supernova designation. The symbols "?" denote an unconfirmed SN and "*" the occurrence of multiple SN discoveries in the same galaxy.

2: parent galaxy identification. In case a galaxy has different identifications, we adopted the following priority: NGC, IC, MCG (M), UGC, ESO (E), PGC, Leda, others. In some cases specific names are reported, e.g. LMC. Anonymous galaxies are listed with the letter A followed by the coordinates. In a few cases, where the association with a definite parent galaxy was not possible, we have filled the field with INTERGALACTIC.

3-4: equatorial coordinates of the parent galaxy at the 2000.0 epoch.

5-6: equatorial coordinates of the supernova at the 2000.0 epoch.

7: morphological type of the parent galaxy.

8: morphological type code for the parent galaxy (coding as in RC3).

9: only for disk-like system, inclination of the polar axis with respect to the line of sight in degrees ( 0 for face on systems).

10: position angle of the major axis of the parent galaxy (North Eastwards) in degrees. 


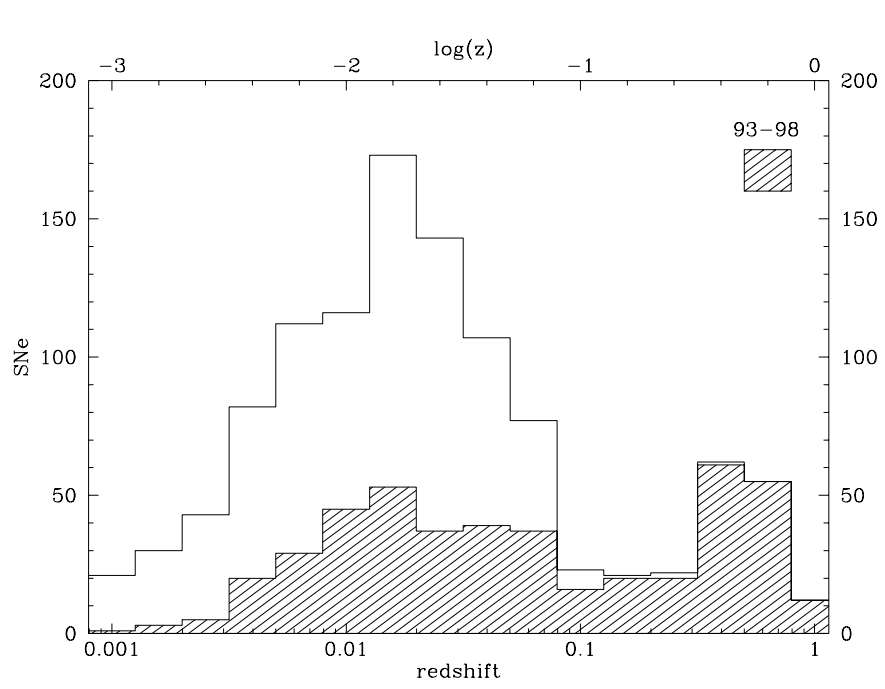

Fig. 2. Distribution of SNe with the redshift of the host galaxy. The shaded area is relative to the $\mathrm{SNe}$ discovered in the last 6 years
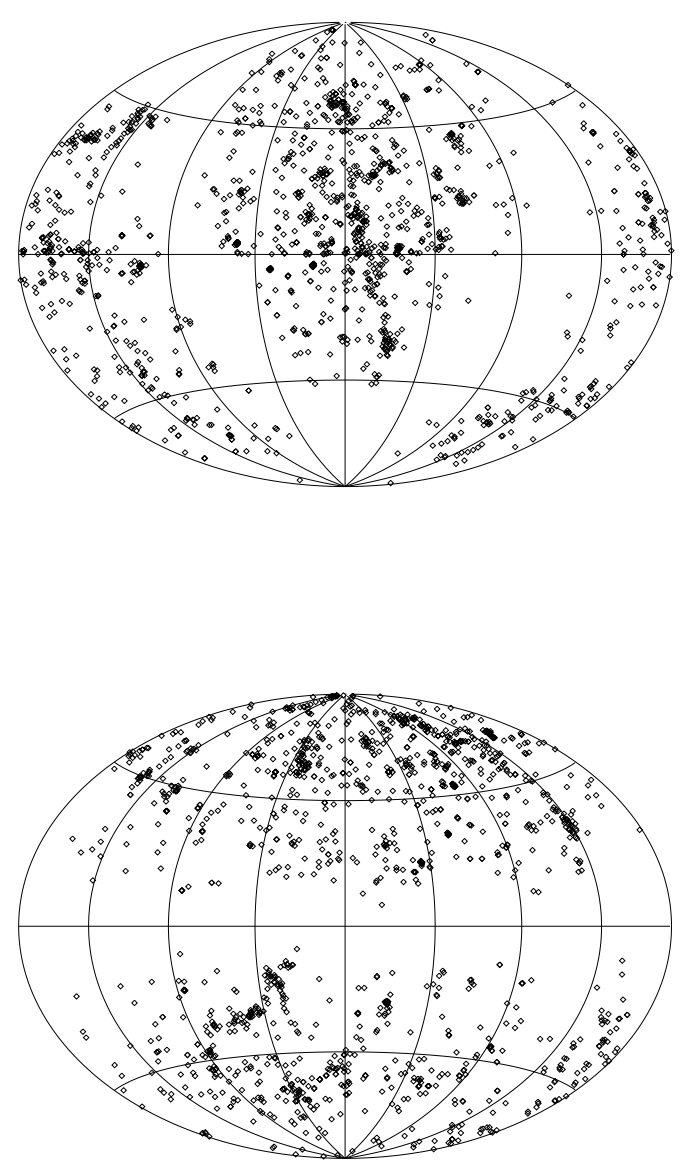

Fig. 3. Distribution of SNe in the sky in equatorial (top) and galactic (bottom) coordinates

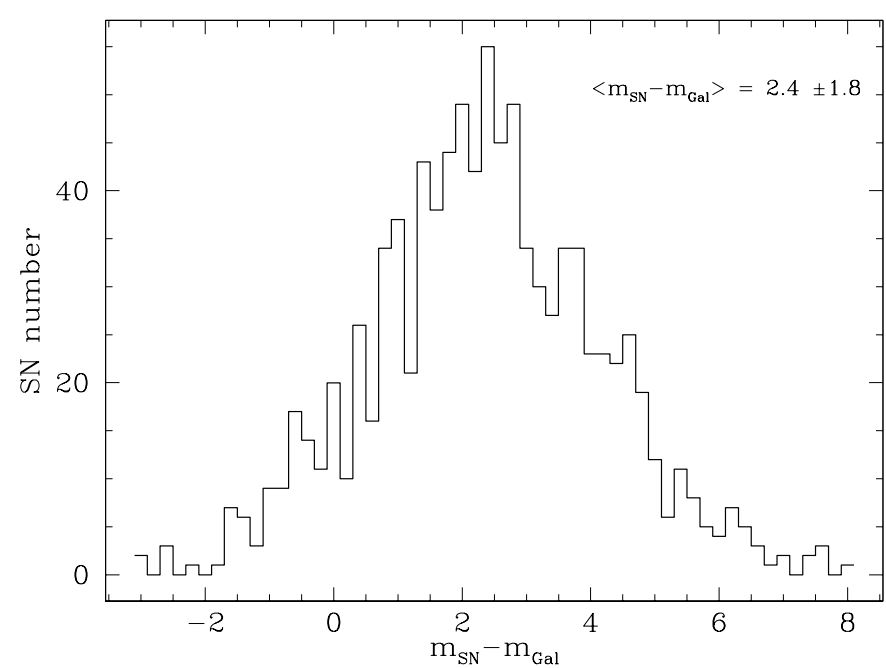

Fig. 4. Histogram of the difference between the SN and galaxy magnitudes

11: heliocentric radial velocity of the parent in $\mathrm{km} \mathrm{s}^{-1}$, but for objects with redshift $z \geq 0.1$ where the $z$ value has been listed.

12: integrated $B$ magnitude of the parent, mostly from the RC3 or LEDA. In a few cases only photographic magnitudes (prefixed by "p") are available.

13: decimal logarithm of the apparent isophotal diameter, in 0.1 arcmin units.

14-15: $\mathrm{SN}$ offset from the galaxy nucleus in arcsec, in the $\mathrm{E} / \mathrm{W}$ and $\mathrm{N} / \mathrm{S}$ direction respectively.

16: if available, supernova magnitude at maximum (photometric band indicated); otherwise discovery magnitude (labelled by "*"). A magnitude without band means that the observation has not been made in a standard photometric system (e.g. those reported in the discovery announcement as photographic, blue plate, red plate, CCD without filter, and so on).

17: supernova type, mostly from spectroscopy. In a few cases, marked by "**", types have been inferred from the light curve.

18: if known, epoch of maximum, otherwise "*" marks date of discovery.

19: name(s) of discoverer(s). For organized search teams the acronyms are given.

\section{Remarks}

A major effort has been devoted in searching the literature for accurate magnitudes, epochs of maximum and for assigning supernova types. For this latter task we are indebted to D. Branch (private communication) who provided us with a list of revised supernova types for many SNe. For the supernovae discovered in the periods from 1989 to 1992 and from 1992 to 1998 we have cross checked our data with those of van den Bergh (1994) and the electronic list supplied by the CBAT, respectively. 
Table 5. Distribution of supernovae according to the morphological types of their parent galaxies

\begin{tabular}{|c|c|c|c|c|c|c|c|c|c|c|c|c|c|c|c|c|c|c|c|}
\hline & $E$ & S0 & $\mathrm{S} 0 / \mathrm{a}$ & $\overline{\mathrm{Sa}}$ & Sab & $\overline{\mathrm{Sb}}$ & Sbc & $\mathrm{Sc}$ & $\overline{S c d}$ & $\overline{\mathrm{Sd}}$ & $\mathrm{Sdm}$ & $\mathrm{Sm}$ & $\bar{S}$ & $\mathrm{I} 0$ & $\mathrm{Im}$ & $\mathrm{I}$ & $\mathrm{Pec}$ & $\mathrm{nc}$ & Total \\
\hline I & 10 & 2 & 5 & 9 & 3 & 8 & 6 & 13 & & 2 & & & 1 & & & & & 18 & 77 \\
\hline Ia & 24 & 31 & 6 & 20 & 13 & 28 & 32 & 35 & 10 & 5 & & 1 & 18 & & & & & 187 & 410 \\
\hline Iapec & 3 & 4 & & & 2 & 1 & 3 & 2 & & 1 & & & 1 & & & & 1 & 1 & 19 \\
\hline $\mathrm{Ib}$ & & & 1 & & 1 & 1 & 1 & 9 & 1 & & & & & & 1 & 1 & & 1 & 17 \\
\hline $\mathrm{Ib} / \mathrm{c}$ & & & & & & & 2 & 2 & & 1 & & 1 & 1 & & & & & 2 & 9 \\
\hline Ic & & & 1 & 1 & 1 & 2 & 3 & 9 & 2 & & & & 4 & & & & & 4 & 27 \\
\hline $\mathrm{Iac}$ & & & & & & 2 & & & & & & & & & & & & & 2 \\
\hline II & & & 2 & 7 & 5 & 32 & 23 & 73 & 12 & 8 & 3 & 1 & 11 & 1 & 2 & 6 & & 56 & 242 \\
\hline IIb & & & & & 1 & & & 2 & 1 & & & 1 & & & & & & & 5 \\
\hline IIn & & & 1 & 1 & & 5 & 4 & 10 & 1 & & & & 1 & & & 2 & & 9 & 34 \\
\hline IIpec & & & & 1 & & 1 & & 4 & & & & & 1 & & & & & 2 & 9 \\
\hline Pec & & & & 1 & 1 & 1 & & 1 & 1 & & & & & & & & & 2 & 7 \\
\hline $\mathrm{nc}$ & 38 & 14 & 15 & 32 & 16 & 62 & 42 & 91 & 7 & 8 & 1 & 6 & 58 & & 2 & 19 & 1 & 177 & 589 \\
\hline Total & 75 & 51 & 31 & 72 & 43 & 143 & 116 & 251 & 35 & 25 & 4 & 10 & 96 & 1 & 5 & 28 & 2 & 459 & 1447 \\
\hline
\end{tabular}

Notes: nc means not classified supernovae and/or galaxies.
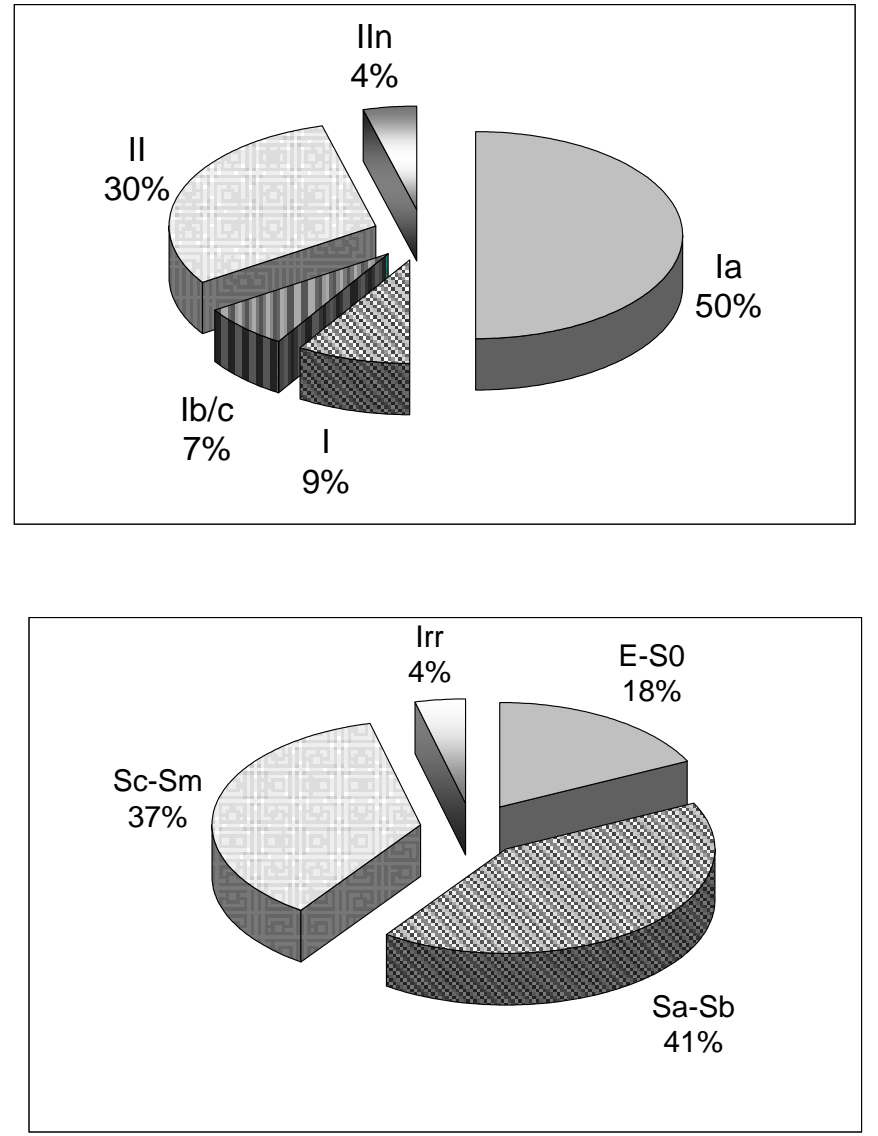

Fig. 5. Distributions of SN types (top) and of the parent galaxy morphological types (bottom)

Discrepancies, in both cases, have been solved by looking at the original literature, mostly consisting of IAU circulars.

The galaxy coordinates are given with various degrees of accuracy depending on the accuracy of the original

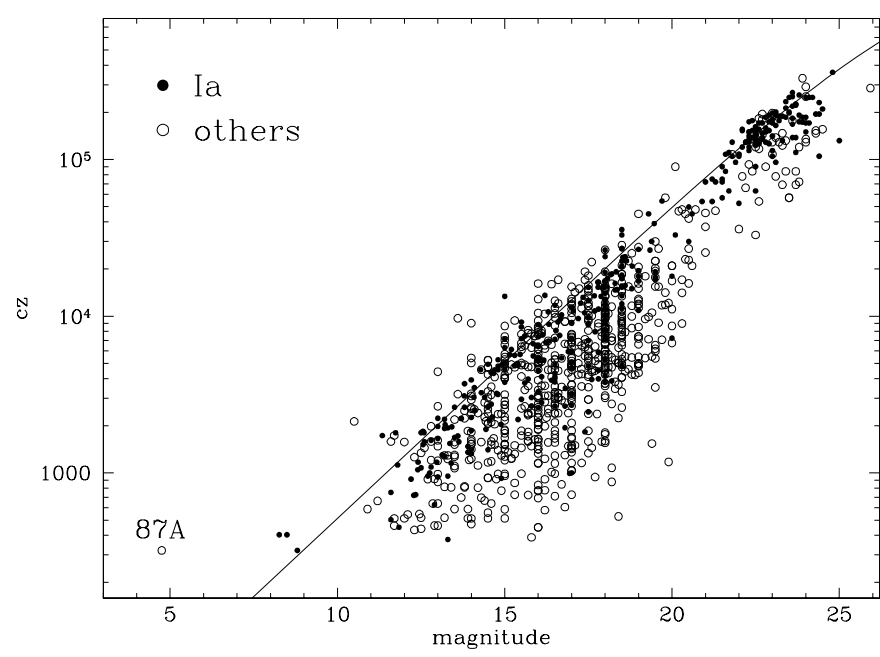

Fig. 6. The Hubble diagram for all the SNe with redshift discovered up to Dec. 31, 1998. The magnitudes are those reported in the Catalogue, i.e. those at maximum when available, or those at discovery. The line is the expected position for "standard" SNIa having $M(\max )=-19.50, H_{0}=65 \mathrm{~km} \mathrm{~s}^{-1} \mathrm{Mpc}, q_{0}=0$

Catalogue. For many anonymous galaxies, in particular for the parent galaxies of high- $z \mathrm{SNe}$, the approximate galaxy position is derived from the supernova coordinates. In some cases, discrepancies may arise between the quoted off-sets of the supernova from the galaxy nucleus and the same data derived from the $\mathrm{SN}$-parent relative coordinates (see e.g. SN 1965C). This happens especially for parent galaxies with ill-defined nuclei. Finally, a few supernovae have been discovered and observed only spectroscopically (e.g. SN 1995bb) and therefore they lack a photometric magnitude.

In Table 3 we list the $37 \mathrm{SNe}$ (excluding SN 1998ab, added in the proofs) which, though announced after the publication of ASC89, were discovered on old plates obtained before December 31, 1988. 
Instead, Table 4 lists the 20 objects which, from the beginning of the searches up to the present time, turned out not to be supernovae. Among these latter objects, SN $1950 \mathrm{E}$ and $1956 \mathrm{C}$ were still included in ASC89. SN 1987G has been deleted because it turned out to be the same object as SN 1987D.

\section{Basic statistics}

In Fig. 1 the productivity of world wide supernova searches through the years is shown. The enormous improvement of the last few years stands out clearly. The dashed area refers to SNe brighter than 14 mag. It must be noted that, despite the renewed effort in SN search, the rate of discovery of bright $\mathrm{SNe}$ is not increasing.

In Fig. 2 we plot the distribution of redshifts and highlight (shaded area) the SNe discovered in the last 6 years. It appears that almost all SNe at redshift $z>0.1$ have been discovered recently and that the recent high- $z$ surveys favour discoveries in the range $0.3<z<0.8$.

In Fig. 3 the sky distribution of the 1447 supernovae both in equatorial (top panel) and galactic (bottom panel) coordinates is shown. Neglecting the avoidance zone defined by the galactic plane, the outcome of the $\mathrm{SN}$ searches of the last years makes the SN sky distributions more homogeneous compared with the same plot reported in ASC89. Overposed to the clustered pattern of the distribution of nearby parent galaxies is evident a smooth background component due to the high- $z$ SNe.

Figure 4 shows the distribution of the difference between the SN and parent galaxy magnitude. The peak of the distribution is at $\left(m_{\mathrm{SN}}-m_{\mathrm{Gal}}\right)=2.4$ with $\sigma=1.8$ which fairly compares with the same result found by Barbon (1968). These numbers may be useful to prepare the strategy of a SN search in given galaxy samples.

Table 5 shows the distribution of supernovae of different types according to the morphological type of their parent galaxies. With respect to Table 3 of ASC89, new SN types are now listed but the overall distribution remains unchanged. Note that the percentage of classified SNe has increased from $40 \%$ to almost $60 \%$ of total discoveries.

Concerning the distribution among different SN types (Fig. 5 top), it turns out that type Ia alone make $50 \%$ of all classified $\mathrm{SNe}$, whereas $\mathrm{Ib} / \mathrm{c}$ are only $7 \%$. The same data of ASC89 give 22\% and 6\%. Actually, in ASC89 a major fraction of type I (54\%) were missing a detailed subtype classification whereas in the present version this is only $14 \%$. On the other hand, the percentage of type II SNe (34\%) remains constant. These numbers show that, since the last decade, the chase for the SNIa is well under way.

The distribution of the parent galaxy morphological types is also shown in Fig. 5 (bottom). It stands out clearly that most $\mathrm{SNe}$ are found in spiral galaxies.

Finally, in Fig. 6 we plot the positions of all SNe with redshift in the Hubble diagram. The line is the expected location for "standard" SNIa having $M(\max )=-19.50$, $H_{0}=65 \mathrm{~km} \mathrm{~s}^{-1} \mathrm{Mpc}, q_{0}=0$. We remark that some SNe, not of type Ia, laying above this line have poor photometry.

Acknowledgements. We are indebted to David Branch for having provided a number of corrections to a previous release of the Catalogue.

\section{References}

Barbon R., 1968, AJ 73, 1016

Barbon R., Cappellaro E., Ciatti F., Turatto M., Kowal T., 1984, A\&AS 58, 735

Barbon R., Cappellaro E., Turatto M., 1989, A\&ASS 81, 421 [ASC89]

Bergh, van den S., 1994, ApJS 92, 219

de Vaucouleurs G., de Vaucouleurs A., Corwin H.G., Buta R.J., Paturel G., Foque P., 1991, Third Reference Catalogue of Bright Galaxies. Springer-Verlag, New York [RC3]

Flin P., Karpowicz M., Murawski W., Rudnicki K., 1979, Acta Cosmologica, part 8

Karpowicz M., Rudnicki K., 1968, Preliminary Catalogue of Supernovae. Warsaw University Press, Warsaw

Kowal C.T., Sargent W.L.W., 1971, AJ 76, 756

Phillips M.M., 1993, ApJ 413, L105

Riess A.G., Press W.H., Kirshner R., 1996, ApJ 473, 88

Saha A., Sandage A., Tammann G.A., Labhardt L., Macchetto F.D., Panagia N., 1999, astro-ph 9904389

Samus N.N., 1995, General Catalogue of Variable Stars, fourth edition, Vol. V. Kosmosinform, Moscow

Sargent W.L.W., Searle L., Kowal C.T., 1974, Supernovae and Supernova Remnants, Cosmovici C.B. (ed). D. Reidel Publ. Dordrecht, p. 33

Zwicky F., 1958, Handbuch der Physik, Flugge S. (ed). Springer-Verlag Berlin, Vol. 51, p. 766

Zwicky F., 1965, Stars and Stellar Systems, Aller L.H. and McLaughlin M.C. (eds). The University of Chicago Press, Vol. VIII, p. 367 


\section{Catalogue}

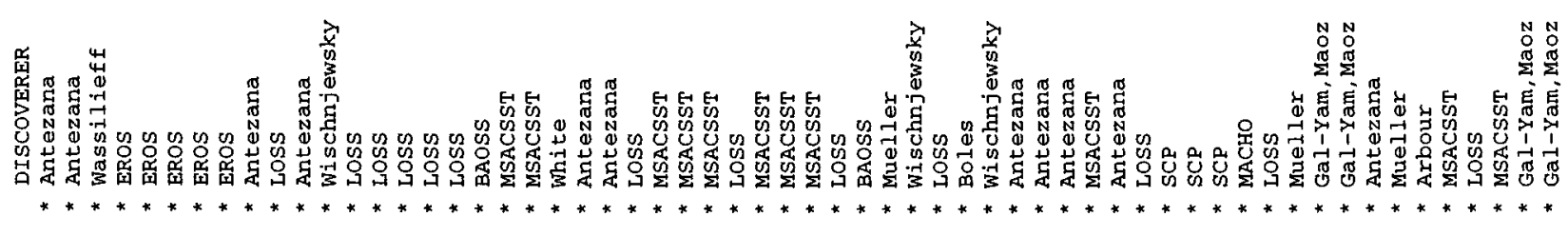

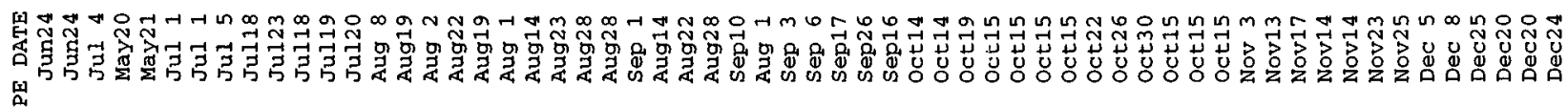

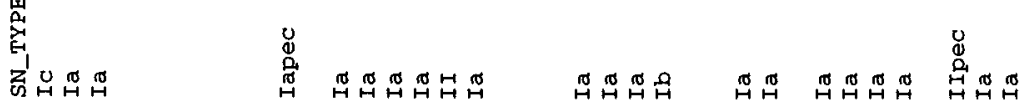

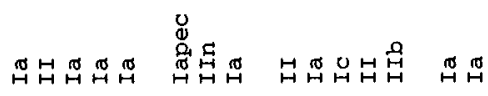

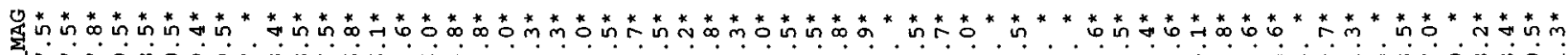

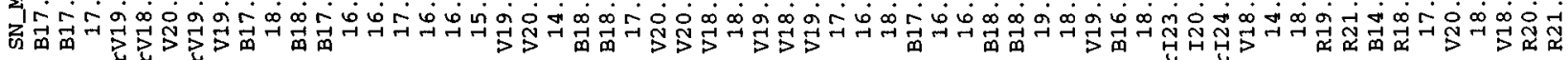

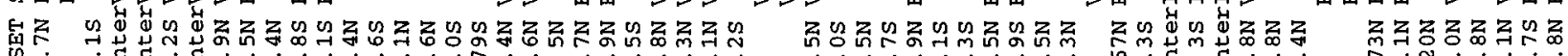
न

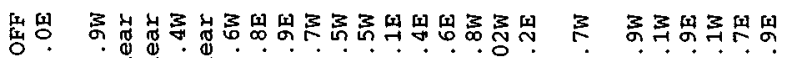

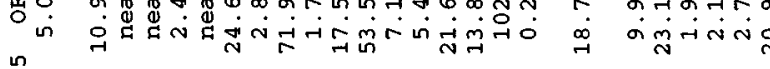

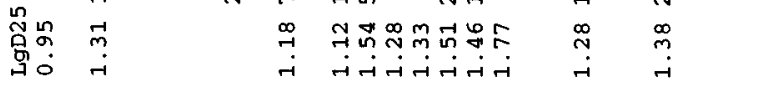

मे

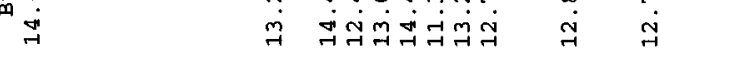

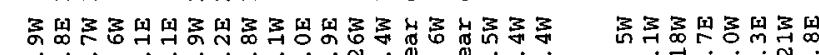

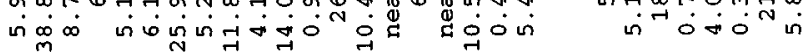

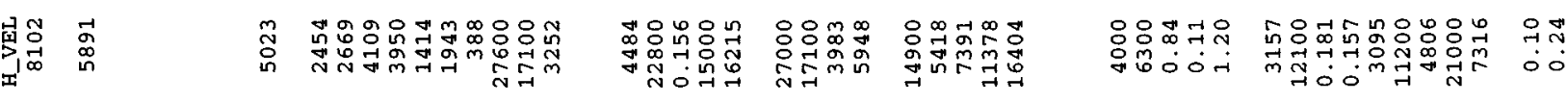

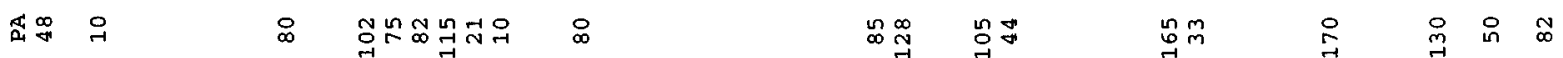

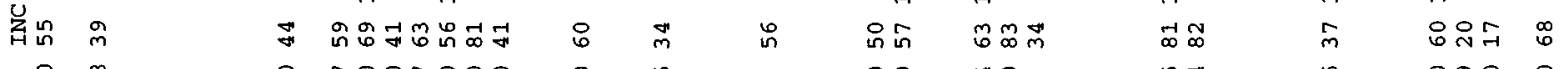

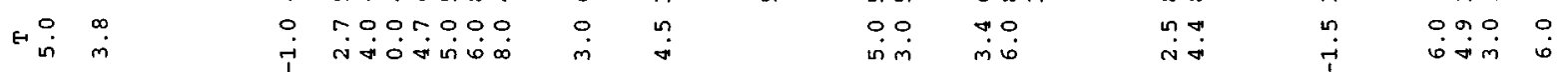

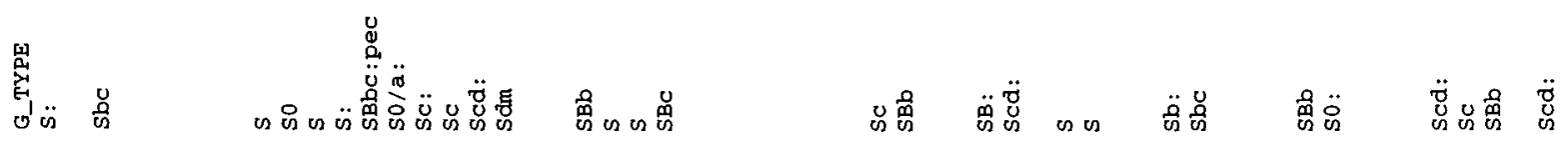

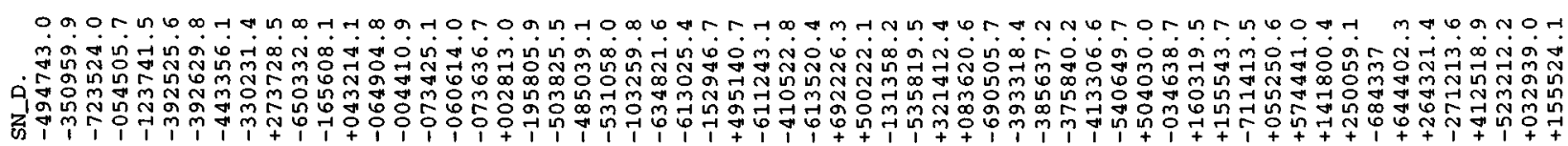

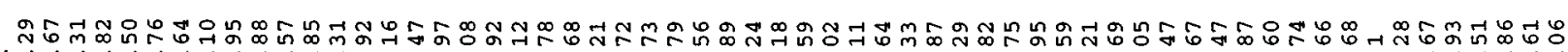

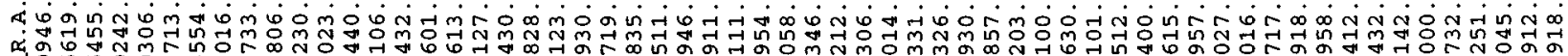

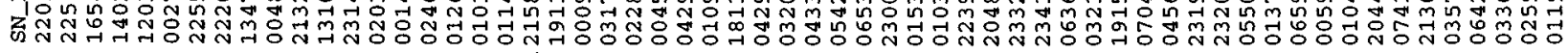

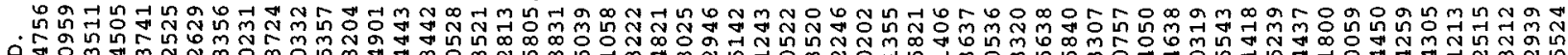

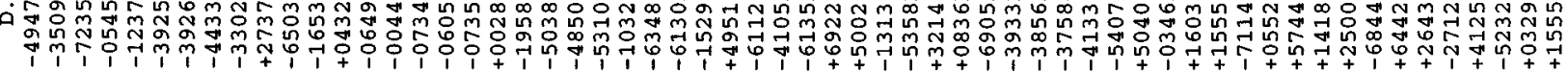

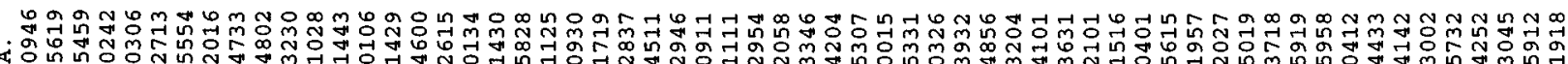

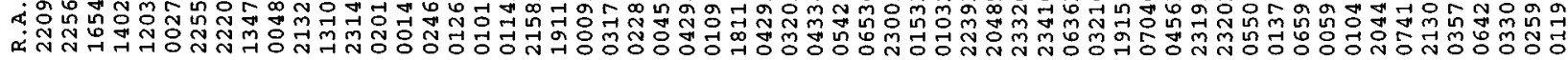

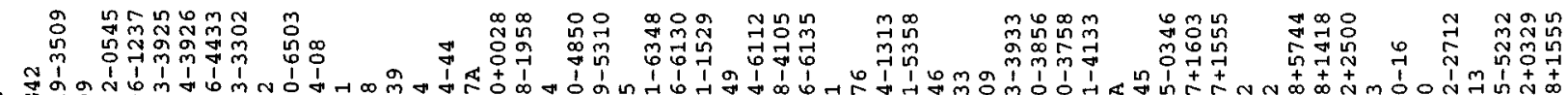

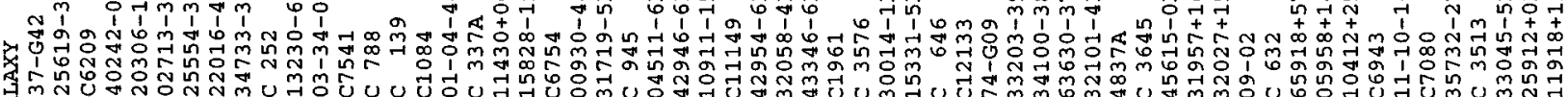

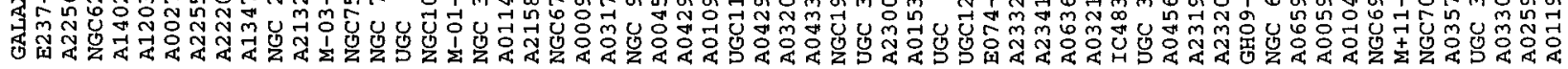

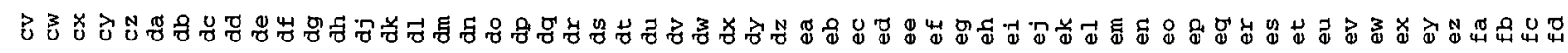

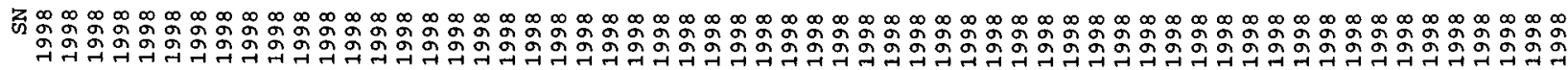

